\title{
Reply To The Article "Bariatric Surgical Practice During the Initial Phase of COVID-19 Outbreak." by Aminian, A., Kermansaravi, M., Azizi, S. et al. Published in Obesity Surgery
}

\author{
Ramon Vilallonga ${ }^{1}$ (D) Ruth Blanco-Colino ${ }^{2} \cdot$ Manel Armengol Carrasco ${ }^{2}$ \\ Published online: 13 May 2020 \\ (C) Springer Science+Business Media, LLC, part of Springer Nature 2020
}

COVID-19 has rapidly spread worldwide since its outbreak in Wuhan, China in December 2019. Pandemic status was declared by World Health Organization in March 2020 [2]. The authors report some of the cases that presented in Iran during the outbreak from 24th February to 4th March. Being the first cases declared from 19th to 25th February in Iran, there could be more patients affected by COVID-19 that could have undergone bariatric elective surgery at that time without being noticed by surgical teams [3]. In fact, the same authors have reported previously a patient who died with morbid obesity, the day before and elective surgery for gastric bypass in Iran [4] .As most of those affected countries in which systematic detection of COVID-19 by doing RT-PCR was not performed, means that we may be seeing the top of the iceberg by reporting only those cases who developed postoperative pneumonia.

The authors highlight that obesity patients are at greater risk in presenting COVID-19 pulmonary complications due to related comorbidities. From the 4 cases included, only the first seems to present with metabolic diseases and respiratory previous pathology. However, usual treatments like 2angiotensin-converting enzyme inhibitors (ACEIs) and angiotensin receptor blockers (ARBs) are not reported in the article. Although further evidence is needed due to controversy, ACEIs and ARBs have been proposed to increase expression of ACE2 and therefore to make patients more susceptible to

Ramon Vilallonga

vilallongapuy@gmail.com

1 Endocrine, Metabolic and Bariatric Unit, Vall d'Hebron Barcelona Hospital Campus, Universitat Autònoma de Barcelona. Center of Excellence for the EAC-BC. Passeig de la Vall d'Hebron 119-129, 08035 Barcelona, Spain

2 General Surgery Department, Vall d'Hebron Barcelona Hospital Campus, Universitat Autònoma de Barcelona. Center of Excellence for the EAC-BC. Passeig de la Vall d'Hebron 119-129, 08035 Barcelona, Spain viral hosting and propagation [4]. Moreover, there are several factors in which obesity can play a role in poor outcomes from COVID-19 infection. Some have proposed that obese patients can present a metabolic dysfunction due to a proinflammatory state and also that adipose tissue can serve as a reservoir for some human viruses [5].

Clinical diagnosis of COVID-19 is heterogeneous among the 4 cases presented. Criteria for performing a chest CT or RT-PCR are not clearly stated. Case 4 misses RT-PCR, while chest $\mathrm{CT}$ has not been done for case 2 . This is a limitation for this early case series that will need to be explored in future perioperative protocols globally [6]. Also, it will be important to take into consideration that some inflammatory parameters such as CRP, white blood cell count, and liver function alterations, as well as pulmonary pneumonia or thromboembolism and collapsed lung, can also be seen in patients presenting with bariatric surgery complications. Therefore, it is important to do a complete differential diagnosis in those patients failing after gastric bypass or sleeve gastrectomy and to include COVID-19 as one more possibility.

The patients included received different treatments that have already been described for COVID-19 pneumonia management, including hydroxychloroquine, antiretroviral (lopinavir and ritonavir), tocilizumab, and hemoperfusion. As there is many controversy and variability in pneumonia management across the globe [7], details on the dose and duration of the treatments should have been included to better use these interesting data in the future.

Finally, short case series can help to have a picture of early experiences in bariatric surgery patients. In the meantime, we need to prepare for when normal activity return to surgical centers. Some recommendations would be needed to be considered with regards to bariatric surgery $[8,9]$. However, it will be key further evidence coming from larger cohort multicentric studies once the elective procedures are rescheduled after COVID-19 pandemic. 
Acknowledgments We thank Aminian $A$ et al. for reporting their experience in bariatric cases during COVID-19 outbreak [1]. We would like to highlight a few points and reservations.

\section{Compliance with Ethical Standards}

Conflict of Interest The authors declare that they have no conflict of interest.

Ethical Approval Statement This article does not contain any studies with human participants or animals performed by any of the authors.

\section{References}

1. Aminian A, Kermansaravi M, Azizi S, et al. Bariatric surgical practice during the initial phase of COVID-19 outbreak. Obes Surg. 2020; https://doi.org/10.1007/s11695-020-04617-x.

2. World Health Organisation(2020) WHO Director-General's opening remarks at the media briefing on COVID-19 - 11 March 2020 [Internet]. Available from: https://www.who.int/dg/speeches/detail/ who-director-general-s-opening-remarks-at-the-media-briefing-oncovid-19\%2D\%2D-11-march-2020

3. Tuite AR, Bogoch II, Sherbo R, et al. Estimation of coronavirus disease 2019 (COVID-19) Burden and potential for international dissemination of infection from Iran. Ann Intern Med. 2020. https://doi.org/10.7326/ M20-0696.

4. Aminian A, Safari S, Razeghian-Jahromi A, et al. COVID-19 outbreak and surgical practice. Ann Surg. 2020;216:1.

5. Patel AB, Verma A. COVID-19 and angiotensin-converting enzyme inhibitors and angiotensin receptor blockers. What is the evidence? JAMA. 2020;11(8):875-9.

6. Kassir R. Risk of COVID-19 for patients with obesity. Obes Rev. 2020;194(March):10-1.

7. Ai T, Yang Z, Hou H, et al. Correlation of chest CT and RT-PCR testing in coronavirus disease 2019 (COVID-19) in China: a report of 1014 cases. Radiology. 2020;26:200642.

8. Chen X, Liu Y, Gong Y, et al. Perioperative management of patients infected with the novel coronavirus: recommendation from the joint task force of the Chinese society of anesthesiology and the Chinese association of anesthesiologists. Anesthesiology 2020. https://doi. org/10.1097/ALN.0000000000003301.

9. Yang W, Wang C, Shikora S, Kow L (2020) Recommendations for metabolic and bariatric surgery during the COVID-19 pandemic from IFSO. Obes Surg [internet]. 2020;1-3. Available from: http:// www.ncbi.nlm.nih.gov/pubmed/32291701

Publisher's Note Springer Nature remains neutral with regard to jurisdictional claims in published maps and institutional affiliations. 